\title{
CLEAR SIGNS
}

\section{Dear Reader,}

At long last, the German government is tackling the problem of "traffic sign clutter". The primary objective of the Federal Ministry of Transport is to thin out the dense jungle of traffic signs in order to raise public acceptance and improve road safety.

From 1 April, six traffic signs will be abolished, including sign 150 of the German driving etiquette called StVO, which shows what looks like a garden fence inside a warning triangle and is meant to warn of a level crossing with a barrier or gate. Some children had actually been misinterpreting this sign as a playground fence. It is being replaced by the sign 151, depicting a modern, oncoming electric train. When I was a child and was waiting at the level crossing with my bicycle, the sign still portrayed a steam locomotive. Although installation of this old sign has not been permitted since 2009, it will remain valid until 2019. Also being deleted are the white arrow (one-way street, 353) and the white number (advisory speed limit, 380) on a blue square as well as sign 388 "Verge not trafficable by motor vehicles with more than two wheels". Signs of this type that have already been installed will remain in force until the end of October 2022. All very commendable.

But in true bureaucratic fashion, while six signs are being deleted, six new ones are being introduced. So much for reducing "sign clutter". The new signs include a controlled parking zone and the end of a diversion or recommended route (all three unnecessary) as well as a sign for a cul-de-sac that is open for cyclists and pedestrians but not for vehicles (more sensible). As always, the motto seems to be "the more, the merrier".
But it is not only the government that is doing something. Local authorities are also becoming active. According to the motorists' organisation ADAC, one third of all traffic signs in towns are superfluous. The mayor of my home town set a good example by going around the town with the ADAC and putting sacks over unnecessary traffic signs. For example, a "no parking” sign in a small cul-de-sac was covered because parking is prohibited anyway in streets less than three metres wide. The blue plastic sacks might look a little strange, but they reveal just how many signs were contributing to our information overload. In this case, less is more.

I find this a useful activity. It helps to combat overstimulation of our senses and raises our awareness of important traffic signs. I hope it is also a reason to pay more attention to other rules of the road.

Best regards,

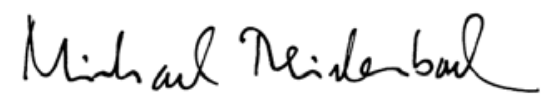

DIPL.-ING. MICHAEL REICHENBACH, Vice Editor-in-Chief Wiesbaden, 17 April 2013

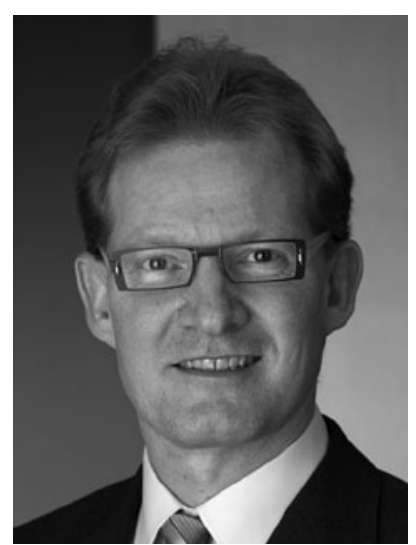

